\title{
Avaliação clínica e ecocardiográfica de pacientes submetidos a implante de bioprótese mitral com preservação da valva natural
}

Altamiro Ribeiro DIAS*, Luiz F. P. ASSUMPÇĀO*, Volnei CASTANHO*, Sérgio Mattos LOMELINO*, Luiz Abba BRITO*, Osmar SAMUEL*, Caio Cesar J. MEDEIROS*, Cesar A. M. CATANI*, José R. PARGA FILHO*, José António CHINELATO*, Adib D. JATENE*

DIAS, A. R.; ASSUMPÇĀO, L. F. P.; CASTANHO, V.; LOMELINO, S. M.; BRITO, L. A.; SAMUEL, O.; MEDEIROS, C. C. J.; CATANI, C. A. M.; PARGA FILHO, J. R.; CHINELATO, J. A.; JATENE, A. D. - Avaliação ecocardiográfica de pacientes submetidos a implante de bioprótese mitral com preservaçăo da valva natural. Rev. Bras. Cir. Cardiovasc., 5(1): 26-34, 1990.

RESUMO: O objetivo deste trabalho é apresentar os resultados pós-operatórios preliminares de 16 pacientes submetidos a implante de bioprótese valvular de pericárdio bovino com preservaçáo de suas valvas naturais, operados sequiencialmente no período de março a setembro de 1989. As cúspides insuficientes foram pregueadas no anel mitral com pontos em $U$ passados nas bordas livres e no anel mitral, sem quaisquer ressecçóes. Foram feitos estudos comparativos no pré e no pós-operatório das seguintes grandezas: diâmetro do átrio esquerdo, diâmetro do ventrículo esquerdo (VE), fração de ejeção e gradiente transvalvares mitrais (pico e médio). A avaliaçăo estatística foi feita através do teste $T$ de Student. 0 valor médio do diâmetro do VE no pré-operatório era de $67,31 \mathrm{~mm}$, caindo para $60,50 \mathrm{~mm}$ no pós-operatório. O valor crítico de $T$ foi 2,131 , sendo o valor observado para a variaçáo do diâmetro de VE igual a 3,18 . O diâmetro de átrio esquerdo (AE) variou de $60,25 \mathrm{~mm}$ para $49,31 \mathrm{~mm}$ com $\mathrm{T}$ igual a 6,72 . O valor médio da fraçāo de ejeção no pré-operatório foi de 0,72 , variando para $0,65 \mathrm{com} T$ igual a 2,68 . Os gradientes (valores médios) transvalvares variaram de $23,7 \mathrm{~mm}$ de $\mathrm{Hg}$ de pico e 12,5 de média, respectivamente, para 9,10 e 5,125. Estes valores residuais são perfeitamente aceitáveis de acordo com a literatura e a própria experiência do Serviço. Não houve complicaçóes ou óbitos na série. Em nenhum paciente ocorreu gradiente na via de saida de VE que pudesse sugerir obstruçăo pela cúspide anterior. Os autores concluem pela eficácia da técnica a qual é de fácil e rápida execuçāo, afasta desinserçōes atrioventriculares, nāo gera gradientes, favorece ótimo desempenho ventricular com rápida e acentuada regressáo dos diâmetros de $A E$ e VE.

DESCRITORES: próteses valvulares cardiacas, biológicas; próteses valvulares cardiacas, cirurgia.

\section{INTRODUÇÃO}

A experiência cirúrgica do Serviço de Cardiologia da Fundação Hospital Ítalo-Brasileiro Umberto I (antigo Hospital Matarazzo) com cirurgia valvar, no período de
4 de novembro de 1986 a 30 de setembro de 1989 , é composta de $\mathbf{4 4 1}$ doentes distribuídos como se vê no Quadro 1.

A mortalidade global desse grupo é de $23(5,21 \%)$ pacientes.

\footnotetext{
Trabalho realizado na Fundaçăo Hospital Italo-Brasileiro Umberto I. Săo Paulo, SP, Brasil.

Apresentado ao 17: Congresso Nacional de Cirurgia Cardiaca. Belo Horizonte, MG, 6 e 7 de abril, 1990.

- Da Fundaçăo Hospital ltalo-Brasileiro Umberto I.

Endereço para separatas: Altamiro Ribeiro Dias. Rua Corrêa Dias, 496. 04104 Săo Paulo, SP, Brasil.
} 
DIAS, A. R.; ASSUMPÇĀO, L. F. P.; CASTANHO, V.; LOMELINO, S. M.; BRITO, L. A.; SAMUEL, O.; MEDEIROS, C. C. J.; CATANI, C. A. M.; PARGA FILHO, J. R.; CHINELATO, J. A.; JATENE, A. D. - Avaliação ecocardiográfica de pacientes submetidos a implante de bioprótese mitral com preservaçăo da valva natural. Rev. Bras. Cir. Cardiovasc., 5(1): 26-34, 1990.

\begin{tabular}{|c|c|c|}
\hline \multicolumn{3}{|c|}{$\begin{array}{c}\text { QUADRO } 1 \\
\text { EXPERIENCIA COM CIRURGIA VALVAR } \\
04 / 11 / 86 \text { a } 30 / 09 / 89\end{array}$} \\
\hline \multicolumn{2}{|c|}{ Procedimento cinúrgico } & N: de casos \\
\hline Substituiçőes isoladas de valvas mitrais naturais (M) & Técnica Habitual & 70 \\
\hline \multicolumn{2}{|c|}{ Reoperaçōes para substituiçōes de próteses mitrais disfuncionantes } & 77 \\
\hline $\begin{array}{l}\text { Comissurotomias com } \\
\text { Resseccōes trapezoida }\end{array}$ & ilarotomia & $\begin{array}{r}116 \\
02\end{array}$ \\
\hline \multicolumn{2}{|c|}{ Experiência global com o tratamento cirúrgico isolado da valva mitral } & 281 \\
\hline & 60 \\
\hline \multicolumn{2}{|c|}{ Reoperaçőes para substituiçōes de próteses aórticas disfuncionantes } & 32 \\
\hline \multicolumn{2}{|c|}{ Comissurotomias e descalcificaçōes de valvas aórticas } & 11 \\
\hline \multirow{2}{*}{\multicolumn{2}{|c|}{$\begin{array}{l}\text { Experiência global com o tratamento cirúrgico isolado da valva aórtica } \\
\text { Duplas trocas }(\mathrm{M} \text { e } \mathrm{AO})\end{array}$}} & 103 \\
\hline & & 24 \\
\hline \multicolumn{2}{|c|}{ Tratamento cirúrgico de tríplice disfunçăo valvar (M, Ao e T) } & 31 \\
\hline \multicolumn{2}{|c|}{ Substituiçōes isoladas da valva tricúspide (Endocardite) } & 02 \\
\hline \multicolumn{2}{|l|}{ Total } & 441 \\
\hline
\end{tabular}

$M=$ mitral; $A 0=$ aórtica; $T=$ tricúspide.

QUADRO 2

SUBSTITUIÇÃO ISOLADA DA VALVA MITRAL NATURAL CAUSAS DE OBITOS

\begin{tabular}{ll}
\hline \multicolumn{1}{c}{ Complicaçōes } & N: de Casos \\
\hline Coagulopatia grave & 2 \\
Sangramento na junção atrioventricular & 1 \\
Rotura do ventrículo esquerdo & 1 \\
Arritmias graves & 2 \\
Síndrome de baixo débito & 2 \\
\multicolumn{1}{c}{ Total } & 8 \\
\hline
\end{tabular}

Analisando-se, especificamente, os pacientes operados devido a afecçōes mitrais, vemos que 77 foram reoperados devido a próteses disfuncionantes, 86 tiveram suas valvas naturais substituídas devido a diferentes lesóes nas quais foram inviáveis procedimentos plásticos. Setenta pacientes tiveram suas valvas substituídas pela técnica convencional, isto é, com ressecção da cúspide anterior, músculos papilares, com ou sem preservaçăo da cúspide posterior. Dezesseis pacientes foram operados pela técnica de implante, a qual descreveremos adiante e que é objeto deste trabalho.

Houve oito $(9,30 \%)$ óbitos nesse grupo de 86 doentes, mortalidade que consideramos elevada.

No Quadro 2, temos enumeradas, sucintamente, as causas de óbitos. Chama a atenção que dois pacientes apresentaram grandes sangramentos relacionados diretamente à substituiçăo valvar (sangramento atrioventricular e rotura do ventrículo esquerdo).

Preocupados com esses fatos, introduzimos, a partir de 1 : de março de 1989 , uma alteração técnica, a qual consiste, basicamente, em implantar o substituto valvar mitral sem ressecção dos componentes da valva lesada a ser substituída. Foram operados, consecutivamente, 16 doentes, no período, com esssa modificação técnica, sem óbitos.

O objetivo deste trabalho é a análise preliminar dos resultados do tratamento cirúrgico desses 16 doentes.

\section{CASUÍSTICA E MÉTODOS}

Foram estudados 16 pacientes operados seqũencialmente, no período de 1 : de março a 30 de setembro de 1989. Nove eram do sexo masculino e sete do feminino. As idades variaram de 16 a 75 anos, com valor médio de 35,3 anos.

Quatro pacientes tinham diagnóstico clínico de dupla disfunçăo valvar, sendo que os demais 12 pacientes apresentavam insuficiência mitral pura. Todos apresentavam hipertensão pulmonar.

Os pacientes receberam indicação cirúrgica, face à intensa repercussão clínica e laboratorial da cardiopatia.

A avaliaçăo cirúrgica intra-operatória revelou ser inviável procedimentos plásticos nessa série de pacientes.

Nos quatro pacientes com fusāo comissural associada à retraçāo central das cúspides (dupla disfunçăo), realizamos secçōes comissurais até o anel mitral, transformando essa lesão em insuficiência valvar. Foram, então, passados pontos em $U$ de fio de Poliester trançado revestido de polibutilato 2-0 nas bordas livres das cúspides e nos correspondentes segmentos do anel mitral, 


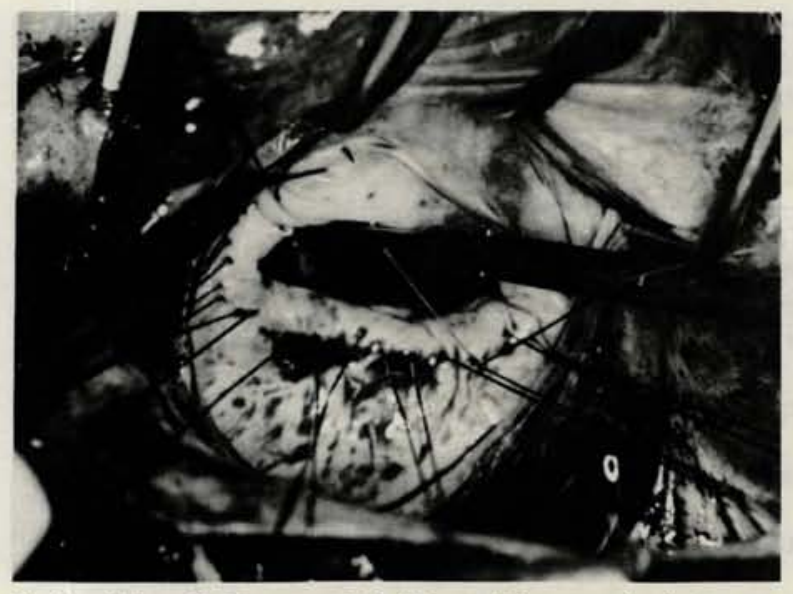

Fig. 1 - Fotografia intra-operatória. Vê-se o átrio esquerdo aberto, sendo passados pontos em U nas bordas das cúspides e no anel, de forma a "enrolar" a cúspide contra $o$ anel. Na parte superior da fotografia vê-se o detalhe da agulha entrando na borda da cúspide anterior e saindo no anel.

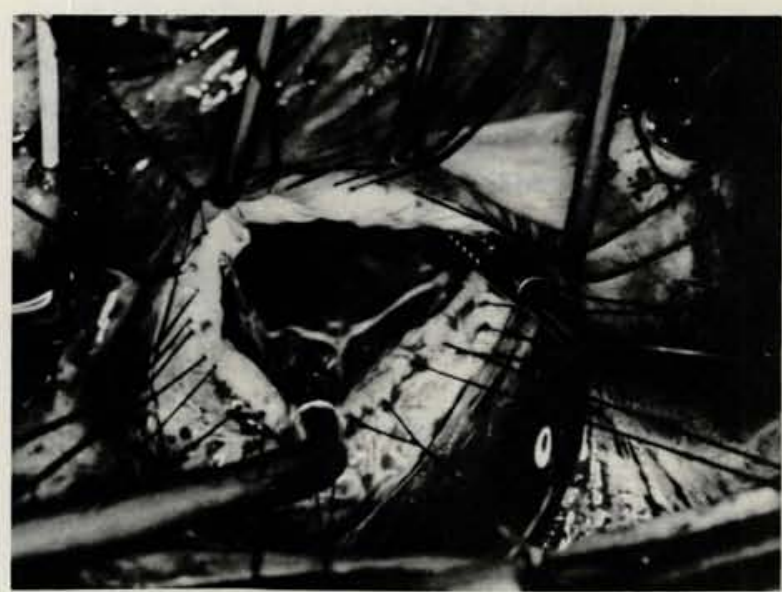

Fig. 2 - Săo vistos todos os pontos passados. No interior do ventrículo esquerdo são visibilizados papilares e cordas.

enrodilhando, desta forma, as cúspides, as quais ficaram totalmente pregueadas e sem se constituir em volume que, eventualmente, pudesse trazer quaisquer dificuldades ao assentamento da prótese. Mesmo em dois pacientes que apresentavam degeneraçăo mixomatosa com cúspides algo redundantes, não se observou qualquer aspecto que pudesse sugerir obstrução da via de saída do ventrículo esquerdo (Figuras 1 e 2 ).

Foram realizadas biopróteses de pericárdio bovino confeccionadas pelos laboratórios Valcor, com diâmetro externo de $29 \mathrm{~mm}$ em um paciente, $31 \mathrm{~mm}$ em nove pacientes e $33 \mathrm{~mm}$ nos seis restantes.

Não houve dificuldade na implantação das próteses após o pregueamento das cúspides.
A avaliação dos resultados foi feita através do estudo clínico dos pacientes no pré e no pós-operatório imediato, bem como da análise comparativa das seguintes grandezas avaliadas ao ecocardiograma: a) diâmetro diastólico do átrio esquerdo (AE); b) diâmetro diastólico do ventrículo esquerdo (VE); c) fraçāo de ejeção; d) gradiente transvalvar mitral (pico e médio); e) pesquisa de gradientes na via de saída do VE.

Foi utilizado o teste $\mathrm{T}$ de Student para a análise estatística.

A avaliação dinâmica ecocardiográfica demonstra, claramente, papilares, cordoalha e cúspides enrodilhadas, sem interferência das estruturas preservadas no desempenho da bioprótese (Figuras 3,4 e 5)

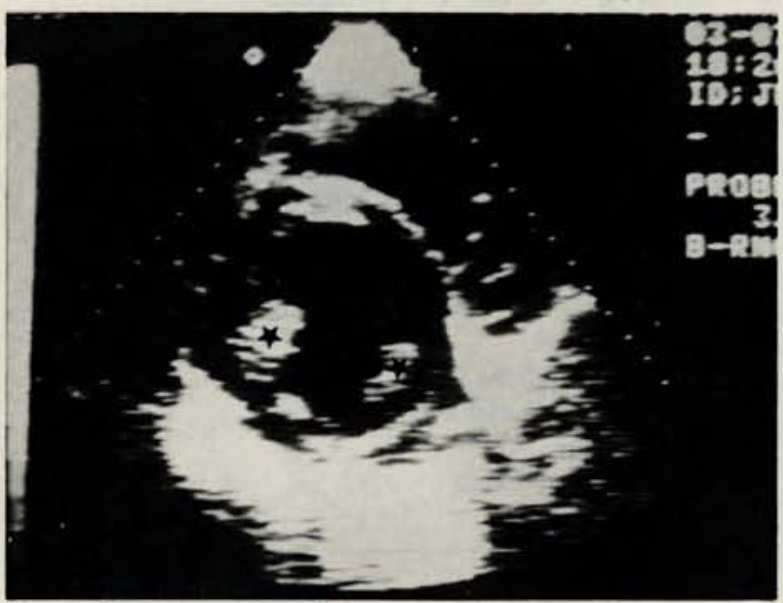

Fig. 3-Corte ecocardiográfico transversal, mostrando os dois papilares.

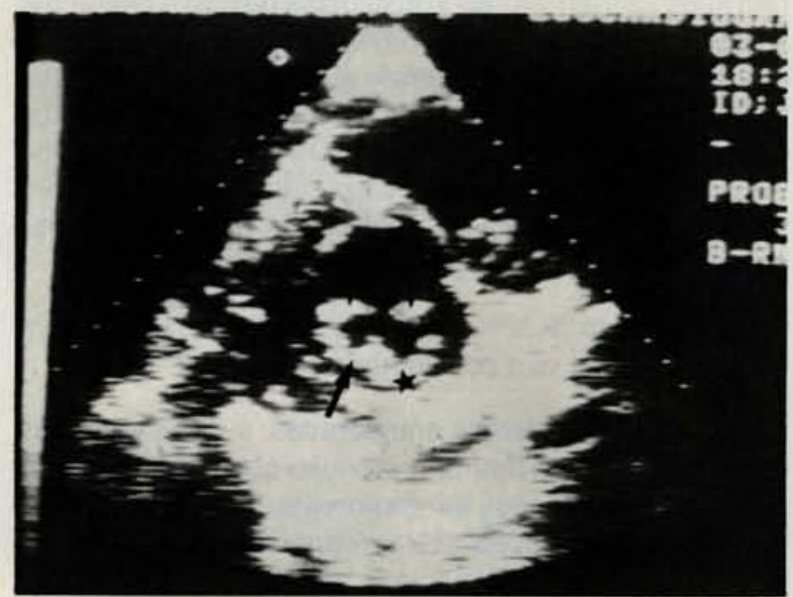

Fig. 4-Corte transversal mais próximo ao anel mitral. As setas apontam para as três hastes da bioprótese implantada. A outra marca está sobre a cúspide enrolada contra o anel mitral. 
DIAS, A. R.; ASSUMPÇÃO, L. F. P.; CASTANHO, V.; LOMELINO, S. M.; BRITO, L. A.; SAMUEL, O.; MEDEIROS, C. C. J.; CATANI, C. A. M.; PARGA FILHO, J. R.; CHINELATO, J. A.; JATENE, A. D. - Avaliação ecocardiográfica de pacientes submetidos a implante de bioprótese mitral com preservação da valva natural. Rev. Bras. Cir. Cardiovasc., 5(1): 26-34, 1990.

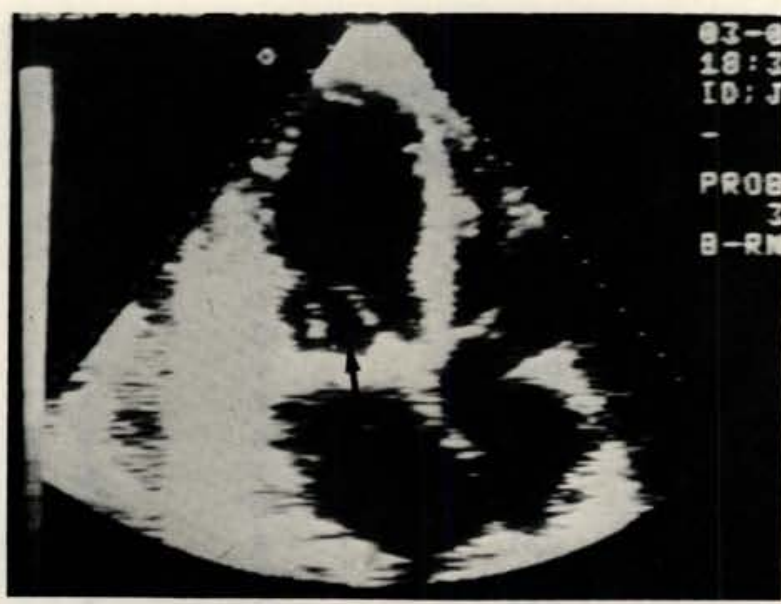

Fig. 5-Corte apical quatro câmaras. Ao lado da seta vêem-se duas das três hastes da bioprótese. Săo vistas também cordas tendineas para o respectivo músculo papilar.

\section{RESULTADOS}

Chamou a atençăo, no pós-operatório imediato, a acentuada e precoce regressăo observada aos $\mathrm{RX}$ da área cardiaca de pacientes que apresentavam cardiomegalia expressiva no pré-operatório, com grande aumento do átrio esquerdo, tal como veremos adiante, no estudo ecocardiográfico.

Nenhum desses pacientes apresentou intercorrências e nāo houve necessidade do emprego de drogas vaso-ativas.

\section{Não houve óbitos na série.}

Verificamos, também, não haver qualquer dificuldade com o tamanho das próteses, as quais apresentaram diâmetros variando entre 31 e $33 \mathrm{~mm}$, respectivamente, em nove e sete pacientes. Esses diâmetros têm sido usados, na nossa experiência, na maioria dos pacientes submetidos a troca de valva mitral pelas técnicas convencionais.

Apresentamos, a seguir, os resultados obtidos dos parâmetros ecocardiográficos.

$\mathrm{Na}$ Tabela 1, encontramos os valores pré-operatórios das variáveis estudadas.

Vemos que o diâmetro diastólico do átrio esquerdo variou de 45 a $74 \mathrm{~mm}$, com valor médio de $60,25 \mathrm{~mm}$. O diâmetro do VE variou de 44 a $89 \mathrm{~mm}$, com valor médio de $67,3 \mathrm{~mm}$. A fração de ejeção variou de 0,52 a 0,84 , com valor médio de 0,71 . O gradiente transvalvar mitral apresentou valor médio de pico de $23,7 \mathrm{mmHg}$ e valor médio de $12,5 \mathrm{mmHg}$.

$\mathrm{Na}$ Tabela 2, encontramos os valores pós-operatórios das variáveis estudadas.

Já no pós-operatório imediato, o diâmetro diastólico do átrio esquerdo variou de 38 a $64 \mathrm{~mm}$, com valor médio de $49,3 \mathrm{~mm}$. O diâmetro do VE variou de 52 a $77 \mathrm{~mm}$, com valor médio de $60,5 \mathrm{~mm}$. A fraçăo de ejeção variou de 0,51 a 0,85 . O valor médio de pico do gradiente transvalvar mitral, no pós-operatório, foi de $9,10 \mathrm{mmHg}$ e a média foi $5,25 \mathrm{mmHg}$. Aplicando-se

TABELA 1

VALORES PRÉ-OPERATÓRIOS

\begin{tabular}{|c|c|c|c|c|c|}
\hline \multirow{2}{*}{ No de Caso } & \multirow{2}{*}{ Diámetro Diastólico do AE } & \multirow{2}{*}{ Diâmetro Diastólico do VE } & \multirow{2}{*}{ Fração de Ejeção } & \multicolumn{2}{|c|}{ Gradientes } \\
\hline & & & & Pico & Médio \\
\hline 01 & 74 & 76 & 0,72 & - & - \\
\hline 02 & 45 & 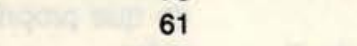 & 0,78 & - & - \\
\hline 03 & 70 & 69 & 0,72 & 22 & 10 \\
\hline 04 & 60 & 58 & 0,78 & 17 & 15 \\
\hline 05 & trat & 72 & 0,79 & 34 & 15 \\
\hline 06 & 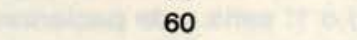 & $\sqrt{12}$ & 0,84 & - & - \\
\hline 07 & 69 & a & 0,76 & 29 & 15 \\
\hline 08 & 56 & 75 & 0,56 & 24 & 12 \\
\hline 09 & 60 & 64 & 0,52 & - & - \\
\hline 10 & 61 & 89 & 0,57 & 19 & 12 \\
\hline 11 & 57 & 77 & 0,73 & 20 & 12 \\
\hline 12 & 64 & 75 & 0,72 & - & - \\
\hline 13 & 47 & 59 & 0,73 & 15 & 07 \\
\hline 14 & 48 & 46 & 0,75 & 34 & 16 \\
\hline 15 & 72 & 44 & 0,68 & - & 11 \\
\hline 16 & 50 & 70 & 0,83 & - & - \\
\hline Valor Médio & 60,25 & 67,3 & 0,71 & 23,7 & 12,5 \\
\hline
\end{tabular}

$A E=$ átrio esquerdo; $V E=$ ventriculo esquerdo. 
DIAS, A. R.; ASSUMPÇÃO, L. F. P.; CASTANHO, V.; LOMELINO, S. M.; BRITO, L. A.; SAMUEL, O.; MEDEIROS, C. C. J.; CATANI, C. A. M.; PARGA FILHO, J. R.; CHINELATO, J. A.; JATENE, A. D. - Avaliação ecocardiográfica de pacientes submetidos a implante de bioprótese mitral com preservaçăo da valva natural. Rev. Bras. Cir. Cardiovasc., 5(1): 26-34, 1990.

TABELA 2

VALORES PÓS-OPERATÓRIOS

\begin{tabular}{|c|c|c|c|c|c|}
\hline \multirow{2}{*}{ Ni de Caso } & \multirow{2}{*}{ Diâmetro Diastólico do AE } & \multirow{2}{*}{ Diametro Diastolico do VE } & \multirow{2}{*}{ Fração de Ejeção } & \multicolumn{2}{|c|}{ Gradientes } \\
\hline & & & & Pico & Médio \\
\hline 01 & 54 & 53 & 0,85 & 7 & 3 \\
\hline 02 & 39 & 52 & 0,64 & 5 & 2 \\
\hline 03 & 64 & 60 & 0,66 & 13 & 5 \\
\hline 04 & 49 & 50 & 0,66 & 9 & 7 \\
\hline 05 & 55 & 72 & 0,62 & 7 & 3 \\
\hline 06 & 49 & 58 & 0,65 & 6 & 3 \\
\hline 07 & 53 & 65 & 0,67 & 7 & 5 \\
\hline 08 & 46 & 75 & 0,54 & 10 & 5 \\
\hline 09 & 44 & 63 & 0,61 & 8 & 5 \\
\hline 10 & 56 & 77 & 0,55 & 10 & 5 \\
\hline 11 & 47 & 63 & 0,58 & 11 & 8 \\
\hline 12 & 52 & 61 & 0,76 & 5,5 & 4,5 \\
\hline 13 & 50 & 62 & 0,51 & 13 & 6 \\
\hline 14 & 44 & 50 & 0,78 & 16 & 7 \\
\hline 15 & 49 & 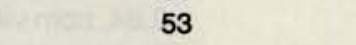 & 0,66 & - & 9,5 \\
\hline 16 & $4=-11$ & 54 & 0,75 & - & 6 \\
\hline Valor Médio & 49,3 & 60,5 & 0,65 & 9,10 & 5,25 \\
\hline
\end{tabular}

$\mathrm{AE}=$ átrio esquerdo; $\mathrm{VE}=$ ventrículo esquerdo.

TABELA 3

TEST T DE STUDENT - VALORES DE T $T C R I T I C O=2.131$

\begin{tabular}{cc}
\hline Grandezas Estudadas & Valores Obtidos de $t$ \\
\hline AE & 6,72 \\
VE & 3,18 \\
FE & 2,68 \\
\hline
\end{tabular}

$\mathrm{AE}=$ átrio esquerdo; $\mathrm{VE}=$ ventrículo esquerdo; $\mathrm{FE}=$ fração de ejeçăo.

o teste T de Student, obteve-se um valor crítico de T igual a 2.131.

Os valores de $\mathrm{T}$ encontrados para as demais variáveis acham-se na Tabela 3.

Vê-se que houve significância nas variaçōes encontradas, especialmente em relaçăo à reduçăo do diâmetro do átrio esquerdo.

Verifica-se ter havido grande significância nas regressōes das câmaras cardiacas, especialmente o átrio esquerdo. do VE.

Não foram encontrados gradientes na via de saída

\section{DISCUSSĀO}

Vários autores ${ }^{1}, 18,20$ chamam a atençāo para a elevada incidência de síndrome de baixo débito associada às substituiçōes valvares mitrais clássicas, especialmente nas primeiras horas do pós-operatório.

A substituição valvar mitral em pacientes com regurgitaçăo crónica provoca redução dos diâmetros do VE e da fração de ejeção (FE) 2, 3, 13, 14, 24, 25 .

A queda da FE parece relacionar-se muito mais à alteração da pós-carga pela retirada do refluxo mitral do que propriamente a alterações do miocárdio ventricular.

Já WONG \& SPOTNITZ ${ }^{25}$ distinguem dois grupos de doentes: a) o 1: seria o de pacientes com diâmetros năo muito aumentados e FE praticamente normal. Nestes, o ecocardiograma demonstra, no pós-operatório, acentuada reduçăo desses diâmetros; b) pacientes com grandes aumentos dos diâmetros do VE e FE já diminuída; nesse grupo, a substituição valvar não promove regressōes do aumento da câmara ventricular, cuja contraçăo depende da redução da pós-carga dada pelo refluxo. Nossos doentes estão todos no 1? grupo.

Todas essas alterações funcionais certamente contribuem para a morbimortalidade das substituiçōes valvares com a técnica de ressecção do aparelho valvar e fixaçăo da prótese. 
DIAS, A. R.; ASSUMPÇĀO, L. F. P.; CASTANHO, V.; LOMELINO, S. M.; BRITO, L. A.; SAMUEL, O.; MEDEIROS, C. C. J.; CATANI, C. A. M.; PARGA FILHO, J. R.; CHINELATO, J. A.; JATENE, A. D. - Avaliação ecocardiográfica de pacientes submetidos a implante de bioprótese mitral com preservação da valva natural. Rev. Bras. Cir. Cardiovasc., 5(1): 26-34, 1990.

RUSHMER et/ alii ${ }^{21}$ estudam a movimentação das cúspides valvares durante o ciclo cardíaco, demontrando que a sua movimentação é restrita pela traçăo exercida sobre elas através das cordas tendíneas. Essa tração mantém as cúspides em aposição parcial durante a diástole e puxa o aparelho valvar em direção à ponta durante a sístole.

Vários trabalhos experimentais ${ }^{9}, 10,11,23$ vieram demonstrar a importância do aparelho valvar mitral na geração de energia pelo ventrículo esquerdo, o qual tem sua funçăo muito deteriorada e de maneira global, quando se interrompe a continuidade ânulo-papilar. Esses trabalhos chamam a atenção para o fato de que a preservaçāo da continuiade entre cúspides, cordas e músculos papilares, seguramente, deve ter efeito positivo em relação à função do VE no pós-operatório de trocas mitrais, especialmente nos ventrículos cronicamente dilatados.

Em 1964, LILLEHEI et alii ${ }^{15}$, relatam sua experiência com 14 doentes submetidos a troca de valva mitral com próteses de Starr-Edwards. Em 13 desses pacientes foi mantida a cúspide posterior e, em um caso, foi mantido todo o aparelho valvar lesado. A incidência de síndrome de baixo débito do Serviço caiu de $37 \%$ para $14 \%$. Os autores frisam a importância da manutenção das cordas tendíneas para o melhor desempenho do VE. A preservação da cordoalha posterior não interferiu com a movimentação da bola das próteses de Starr-Edwards que foram utilizadas.

O conceito criado por LILLEHEI et alii ${ }^{15}$, em 1964, foi esquecido até 1983 , quando HETZER et alii ${ }^{12}$ apresentam 46 pacientes operados com preservação da cúspide posterior e respectivas cordas. Os autores enfatizam a melhoria obtida no próprio Serviço de Hannover face à evolução clínica favorável desses pacientes, os quais praticamente prescindiram de drogas vaso-ativas no pós-operatório, não tendo ocorrido óbito na série. Atribuem tais resultados à mudança de técnica que, até então, vinham usando, ou seja, a ressecçăo de todo o aparelho valvar.

Porque se passaram tantos anos para que a idéia de LILLEHEI et alii ${ }^{15}$ retornasse à prática?

É possivel que o trabalho experimental de RASTELLI et alli ${ }^{19}$, publicado em 1967, afirmando que a preservaçăo da cordoalha e cúspides não tinha qualquer interferência no desempenho ventricular de cães submetidos a substituiçōes mitrais por próteses de Starr-Edwards, tenha tido, na época, uma influência negativa para a aceitação da idéia de LILLEHEl et alii ${ }^{15}$.

Outro fator que talvez tenha contribuido no retardamento da aceitação da idéia é o possível receio dos cirurgiōes de que a preservação das cúspides e cordas pudesse, de alguma forma, interferir com a excursão da bola ou do disco das próteses então utilizadas.
Ainda em 1983, o conceito da preservação das cordas e músculos papilares toma novo impulso, com o relato de DAVID et alii ${ }^{7}$, que propōem a ressecção parcial da cúspide anterior com a preservação das suas cordas tendíneas. Relatam seis pacientes operados dessa forma, nos quais os resultados clínicos foram muito bons e o cateterismo mostrou queda da pressão diastólica final do VE no pós-operatório, bem como melhoria da fração de ejeção.

DAVID et alii ${ }^{6}$ relatam, em 1984, a experiência do Serviço, comparando dois grupos de pacientes submetidos a substituiçáo da valva mitral. Um grupo de 15 pacientes foi operado com a técnica clássica; o outro, de 12 pacientes, foi operado com a preservação das cordas tendíneas. A avaliação funcional foi feita através de estudos radio-isotópicos, sendo que os dados pré-operatórios eram superponiveis. Concluem pelo melhor desempenho funcional nos pacientes onde as cordas tendíneas e músculos papilares são preservados.

DAVID ${ }^{5}$ valoriza tanto a preservação da continuidade ânulo-ventricular, que chega a comparar a substituição valvar mitral com preservação das cordas e músculos papilares, a uma correção de insuficiência valvar pelo procedimento plástico. Enfatiza que a prótese implantada mimetiza as cúspides normais, enquanto que as cordas e papilares preservados colaboram com a função do miocárdio na sístole e na diástole.

Observando os mesmos princípios, MIKI et alii ${ }^{16}$ propöem uma variante técnica, na qual a cúspide anterior é seccionada em duas metades. Cada uma delas é rodada em direção à comissura com a qual se relaciona e aí é fixada.

A cúspide posterior é seccionada no centro da borda livre até próximo ao anel para facilitar a inclusăo da prótese.

Utilizam próteses St. Jude e relatam excelentes resultados clínicos e hemodinâmicos. DINKHUYSEN ${ }^{8}$ entre nós, em recente revisăo, chama a atençăo para os conceitos aqui expostos, ressaltando a importancia da preservação do aparelho subvalvar nas trocas mitrais.

Entendemos que, sempre que a insuficiência mitral é acentuada, com grandes retraçōes das cúspides, torna-se muito fácil a inclusão de uma bioprótese com a técnica descrita e sem qualquer ressecção. Não vemos razōes para ressecçōes completas ou parciais da cúspide anterior, conforme proposto por HETZER et alii ${ }^{12}$, DAVIS et alii ${ }^{6,7}$, ou MIKI et alii ${ }^{16}$.

O pregueamento das cúspides, mesmo que mixomatosas, no anel com pontos em $U$ passados na borda livre e no anel, é suficiente para enrodilhar o que restou da cúspide acometida pelo processo patológico e deixar livre como que um canal na região atrioventricular no 
DIAS, A. R.; ASSUMPÇĀO, L. F. P.; CASTANHO, V.; LOMELINO, S. M.; BRITO, L. A.; SAMUEL, O.; MEDEIROS, C. C. J.; CATANI, C. A. M.; PARGA FILHO, J. R.; CHINELATO, J. A.; JATENE, A. D. - Avaliação ecocardiográfica de pacientes submetidos a implante de bioprótese mitral com preservaçāo da valva natural. Rev. Bras. Cir. Cardiovasc., 5(1): 26-34, 1990.

qual se encaixa, perfeita e confortavelmente, substituto valvar.

Assinale-se que usamos próteses de diâmetros 29 $\mathrm{mm}$ em um doente, $31 \mathrm{~mm}$ em nove e $33 \mathrm{~mm}$ nos seis restantes, sem qualquer dificuldade para a implantaçāo. Vantagem complementar da técnica é a profilaxia de acidentes, tais como a desinserção atrioventricular, a qual é fatal em 40 a $100 \%$ dos casos ${ }^{22}$. O método não é isento de complicaçōes potenciais.

COME et alii ${ }^{4}$ relatam o caso de paciente no qual foi implantada bioprótese porcina de diâmetro de $33 \mathrm{~mm}$ que provocou estenose da via de saída do VE, dada a redundância das estruturas preservadas. Já MOK et alii ${ }^{17}$ relatam dois casos fatais de substituiçōes valvares por próteses de disco do fluxo central, nas quais houve rotura de papilares e impactamento dos discos nas estruturas preservadas, impedindo as próteses de funcionarem.
Entendemos que ambas as complicações possivelmente decorreram de falhas técnicas. A primeira, por inadequado pregueamento das cúspides. A segunda, por excessiva tração das cordas, o que, possivelmente, levou à distensāo, necrose e rotura dos papilares.

Não houve qualquer disfunção das próteses em nossos casos, observável até o presente.

A redução dos diâmetros de átrio e ventrículo esquerdos foi muito acentuada e precoce, analogamente à descrita na literatura $2,3,13,14,24,25$. A redução observada nas fraçōes de ejeçāo, entendemos que deva ser decorrente muito mais do aumento da pós-carga pela eliminação do refluxo mitral do que pela disfunção miocárdica. Limitação potencial ao emprego da técnica será a ocorrência de calcificaçōes extensas infiltrando o anel valvar. Nessas condiçōes, caso não seja possível a adequada remoçăo das calcificaçōes, tornar-se-á dificil enrodilhar as cúspides.

RBCCV 44205-104

DIAS, A. R.; ASSUMPÇÃO, L. F. P.; CASTANHO, V.; LOMELINO, S. M.; BRITO, L. A.; SAMUEL, O.; MEDEIROS, C. C. J.; CATANI, C. A. M.; PARGA FILHO, J. R.; CHINELATO, J. A.; JATENE, A. D. - Clinical and echocardiographic evaluation of patients submitted to mitral bioprosthesis implantations with preservation of their native valves. Rev. Bras. Cir. Cardiovasc., 5(1): 26-34, 1990.

ABSTRACT: The preliminary postoperative results in 16 patients submitted to valvular replacement utilising bovine pericardial valvular bioprostheses, without resection of their natural cusps, are presented. These patients were operated on sequentially from March to September 1989. The incompetent cusps were sutured to the mitral annulus with $U$ stitches placed at their free edges. Pre and postoperative evaluation of the following parameters were obtained: left atrial diameters, left ventricular diameters, ejection fraction and mitral transvalvular gradients (peak and mean). Statistical evaluation utilising the Student's $T$ test was performed. Preoperative mean value of the left ventricular diameter was $67.31 \mathrm{~mm}$, decreasing to 60.50 $\mathrm{mm}$ in the postoperative evaluation. Critical value of $T$ was 2.131 , the value observed for the left ventricle variation being 3.18. The left atrial diameter varied from $60.25 \mathrm{~mm}$ to $49.31 \mathrm{~mm}$ (T equal to 6.72). Preoperative ejection fraction mean value was 0.72 and in the postoperative was $0.65(T=2.68)$. Mean transvalvular gradients varied from $23.7 \mathrm{~mm}$ (peak) and $12.5 \mathrm{~mm}$ (mean) to 9.10 and 5.125 respectively. These residual values are acceptable according to the current literature and the experience of our Institution. There were no complications or deaths in the present series. In no patient gradients in the left ventricular out flow tract suggesting obstruction by the anterior cusp were observed. The authors consider it an efficient technique, easily and rapidly performed, without danger of atrioventricular desinsertions and without significant gradients. Ventricular performance is enhanced and the left atrial and left ventricular diameters decrease considerably.

DESCRIPTORS: heart valves prosteses, biologic; heart valves prostheses, surgery.

\section{REFERÊNCIAS BIBLIOGRÁFICAS}

1 AUSTEN, W. G.; CORNING, H. B.; MORAN, J. M.; SANDERS, C. A.; SCANNELL J. G. - Cardiac emodynamics immediately following mitral valve surgery. J. Thorac. Cardiovasc. Surg., 51: 468-473, 1966.

2 BOUCHER, C. A.; BINGHAM, J. B.; OSBAKKEN, M. D.; OKADA, R. D.; STRAUSS, H. W.; BLOCK, P. C.; LEVINE, F. H.; PHILLIPS, H. R.; POHOST, G. M. - Early changes in left ventricular size and function after correction of left ventricular volume overload. $A m$. J. Cardiol., 47: 991-1004, 1981.

3 BURGGRAF, G. W. \& CRAIGE, E. - Echocardiographic of left ventricular wall motion and dimensions after valvular heart surgery. Am. J. Cardiol., 35: 473-480, 1975.

4 COME, P. C.; RILEY, M. F.; WEINTRAUB, R. M.; WEI, J. Y.; MARKIS, J. E.; LORELL, B. H.; GROSSMAN 
DIAS, A. R.; ASSUMPÇĀO, L. F. P.; CASTANHO, V.; LOMELINO, S. M.; BRITO, L. A.; SAMUEL, O.; MEDEIROS, C. C. J.; CATANI, C. A. M.; PARGA FILHO, J. R.; CHINELATO, J. A.; JATENE, A. D. - Avaliação ecocardiográfica de pacientes submetidos a implante de bioprótese mitral com preservaçăo da valva natural. Rev. Bras. Cir. Cardiovasc., 5(1): 26-34, 1990.

W. - Dynamic left ventricular outflow tract obstruction when the anterior leaflet is retained at prosthetic mitral valve replacement. Ann. Thorac. Surg., 43: 561-563, 1987.

5 DAVID, T. E. - Mitral valve replacement with preservation of chordae tendinae: rationale and technical considerations. Ann. Thorac. Surg., 41: 680-682, 1986.

6 DAVID, T. E.; BURNS, R. J.; BACCHUS, C. M.; DRUCK, M. N. - Mitral valve replacement for mitral regurgitation with and without preservation of chordae tendinae. $J$. Thorac. Cardiovasc. Surg., 88: 718-725, 1984.

7 DAVID, T. E.; UDEN, D. E.; STRAUSS, H. D. - The importance of the mitral apparatus in left ventricular function correction of mitral regurgitation. Circulation, 68 (Supl. 2): 76-82, 1983.

8 DINKHUYSEN, J. J. - O papel das estruturas subvalvares nos implantes de protese mitral. Atual. Cardiol., (SOCESP), 2: 10-12, 1989.

9 HANSEN, D. E.; CAHILL, P. D.; DE CAMPI, W. M.; HARRISON, D. C.; DERBY, G. C.; MITCHELL, R. S.; MILLER, D. C. - Valvular-ventricular interaction: importance of the mitral apparatus in canine left ventricular systolic performance. Circulation, 73: 1310-1320, 1986.

10 HANSEN, D. E.; CAHILL, P. D.; DERBY, G. C.; MILLER, D. C. - Relative contributions of the anterior and posterior mitral chordae tendinae to canine global left ventricular systolic function. J. Thorac. Cardiovasc. Surg., 93: 45-55, 1987.

11 HANSEN, D. E.; SARRIS, G. E.; NICZYPORUK, M. A.; DERBY, G. C.; CAHILL, P. D.; MILLER, D. C. - Phisiologic role of the mitral apparatus in left ventricular regional mechanics, contraction synergy, and global systolic performance. J. Thorac. Cardiovasc. Surg., 97: 521-533, 1989.

12 HETZER, R.; BOUGIOUKAS, G.; FRANZ, M.; BORST, H. G. - Mitral valve replacement with preservation of papillary muscles and chordae tendinae. Revival of a seemingly forgotten concept: I. Preliminary clinical report.

J. Thorac. Cardiovasc. Surg., 31: 291-296, 1983.

13 HUIKURI, H. V. - Effect of mitral valve replacement on left ventricular function in mitral regurgitation. $B r$. Heart J., 49: 328-333, 1983.

KENNEDY, J. W.; DOCES, J. G.; STEWART, D. K. Left ventricular function before and following surgical treatment of mitral valve disease. Am. Heart J., 97: 592-598, 1979.

15 LILLEHEI, C. W.; LEVY, M. J.; BONNABEAU, R. C. Mitral valve replacement with preservation of papillary muscle and chordae tendineae. J. Thorac. Cardiovasc. Surg., 47: 532-543, 1964.

16 MIKI, S.; KUSUHARA, K.; UEDA, Y.; KOMEDA, M.; OHKITA, Y.; TAHATA, T. - Mitral valve replacement with preservation of chordae tendinae and papillary muscle. Ann. Thorac. Surg., 45: 28-34, 1988.

17 MOK, C. K.; CHIU, C. S. W.; CHEUNG, D. L. C.; AUNGKHIN, M. - An unusual lethal complication of preservation of chordae tendineae in mitral valve replacement. J. Thorac. Cardiovasc. Surg., 95: 534-536, 1988.

18 RASTELLI, G. C. \& KIRKLIN, J. W. - Hemodynamic state early after prosthetic replacement of mitral valve. Circulation, 34: 448-461, 1966.

19 RASTELLI, G. C.; TSAKIRIS, A. G.; FRYE, R. L.; KIRKLIN, J. W. - Exercise tolerance and hemodynamic studies after replacement of canine mitral valve with and without preservation of chordae tendinae. Circulation, 35 e 36 (Supl. 1): 34-41, 1967.

20 ROULEAU, C. A.; FRYE, R. L.; ELLIS, F. H. - Hemodynamic state after open mitral valve replacement and reconstruction. J. Thorac. Cardiovasc. Surg., 58: 870-878, 1969.

21 RUSHMER, R. F.; FINLAYSON, B. L.; NASH, A. A. Movements of the mitral valve. Circ. Res., 4: 337-342, 1956.

22 SALOMON, M. N.; HALEVI, A.; SCHUCHMAN, E.; LEVY, M. J. - Left ventricular rupture after mitral valve replacement. Scand. J. Thorac. Cardiovasc. Surg., 15: 235-238, 1981.

23 SALTER, D. R.; PELLOM, G. L.; MURPHY, C. E.; BRUNSTING, L. A.; GOLDSTEIN, J. P.; MORRIS III, J. M.; WECHSLER, A. S. - Papillary-annular continuity and left ventricular systolic function after mitral valve replacement. Circulation, 74 (Supl. 1): 121-129, 1986.

24 SCHULER, G.; PETERSON, K. L.; JOHNSON, A.; FRANCIS, G.; DENNISH, G.; UTLEY, J.; DAILY, P. O.; ASHBURN, W.; ROSS, J. - Temporal response of left ventricular performance to mitral valve surgery. Circulation, 59: 1218-1231, 1979.

25 WONG, C. Y. H. \& SPOTNITZ, H. M. - Systolic and diastolic properties of the human left ventricle during valve replacement for chronic mitral regurgitation. $A m$. J. Cardiol., 47: 40-50, 1981.

\section{Discussão}

DR. JORGE ALBERTAL

Buenos Aires, Argentina

Los resultados hellados en la evolución de diámetros ventriculares, de aurícula isquierda, fracción de eyección y gradientes son los habituales para cualquier procedimento que corrija la insuficiencia mitral (siempre que 
DIAS, A. R.; ASSUMPÇĀO, L. F. P.; CASTANHO, V.; LOMELINO, S. M.; BRITO, L. A.; SAMUEL, O.; MEDEIROS, C. C. J.; CATANI, C. A. M.; PARGA FILHO, J. R.; CHINELATO, J. A.; JATENE, A. D. - Avaliação ecocardiográfica de pacientes submetidos a implante de bioprótese mitral com preservaçāo da valva natural. Rev. Bras. Cir. Cardiovasc., 5(1): 26-34, 1990.

el grupo de pacientes fuera exclusivamente de insuficiência mitral, dato que no se menciona). Solo así se justificaria la caída significativa de la fracción de eyección debido a la restauración de impedancia. Si hubiera pacientes con estenosis mitral pura o predominante, la caída de fracción de eyección tendría un significado negativo asociado a la morbilidad quirurgica. Crremos que los que se pretende hipoteticamente demonstrar al preservar el tejido valvar es obtener una mejor función ventricular residual, comparado con pacientes a quienes se reseca las valvas y aún los pilares. Seria más deseable entonces, tener un grupo control en ese aspecto. Estos datos solo parecen demonstrar que la preservación no es peor que la resección, en cuanto a la función ventricular y que esta técnica no parece generar obstrucción en los flujos transprotésicos, ni en el tracto de salida de ventrículo izquierdo. La literatura mundial tiene trabajos que han demonstrado que la diferencia, conservado las valvulas, se establece especialmente en el ejercicio. El gasto cardiaco aumenta mas que en los pacientes sin conservacion del aparato valvular.

\section{DR. RIBEIRO DIAS (Encerrando)}

Queremos salientar que esta é uma experiência inicial que reune os primeiros 16 pacientes operados por esta variante técnica. Nenhum paciente apresenta estenose mitral. Esta é, justamente, uma limitante à manutenção da valva natural, pois as valvas são, geralmente, intensamente calcificadas, sendo inviável o seu "pregueamento" no anel. Todos os pacientes eram reumáticos e, nos 12 com insuficiência pura, havia intensa retração da cúspide anterior, o que inviabilizou procedimentos conservadores e facilitou muito a preservação do resquício de cúspide anterior. Talvez seja por este fato que não ocorreram gradientes na via de saída do ventrículo esquerdo. Acha-se em implantação protocolo de avaliação dos resultados tardios com provas funcionais que permitam a avaliação do desempenho ventricular no exercício. Tais resultados serăo objeto de apresentação futura. Agradecemos os valiosos comentários e as sugestōes do Dr. Albertal. 